\title{
Damped Vibrations of Rectangular Plate of Variable Thickness Resting on Elastic Foundation: A Spline Technique
}

Robin $^{1 *}$ and Rana US ${ }^{2}$

${ }^{1}$ Department of Applied Science, Quantum School of Technology, Roorkee, India

${ }^{2}$ Department of Mathematics, D.A.V. College, Dehradun, India

\begin{abstract}
In the present paper damped vibrations of homogeneous rectangular plate of linearly varying thickness resting on elastic foundation has been studied. Following Lévy approach, the equation of motion of plate of varying thickness in one direction is solved by quintic spline method. The effect of damping, elastic foundation and taperness is discussed with permissible range of parameters. The frequency parameter $\Omega$ decreases as damping parameter $D_{k}$ increases and it decreases faster in simply supported as compared to clamped-clamped boundary conditions in case of damping parameter and reverses in case of taperness.
\end{abstract}

Keywords: Taperness; Elastic foundation; Damping; Isotropic

\section{Introduction}

In the recent past, it has been observed that the research in the field of vibration is unceasingly accruing immense importance in the modern science, due to the significant role in every field of applied sciences. As fundamental structural elements, plates of various geometries are widely used in various engineering fields such as, aerospace technology, missile technology, naval ship design and telephone industry etc. Due to the appropriate variation of plate thickness, these plates provide the advantage of reduction in weight and size, and also have significantly greater efficiency for vibrations as compared to the plate of uniform thickness. Thus the vibration characteristics of plates having variable thickness have attracted the interest of researchers. An extensive survey of literature up to 1985 on linear vibration of isotropic/anisotropic plates of various geometries has been given by Leissa in his monograph and in a series of review articles [1]. Later on the studies of rectangular plate with uniform/non-uniform thickness has been carried out by many researchers. As space technology is growing rapidly, the importance of study of vibration is increasing; Gupta and Lal [2] have studied the transverse vibrations of a rectangular plate of exponentially varying thickness resting on an elastic foundation by using quintic spline technique. In reality all the vibrations are damped vibration, as free vibrations are ideal and can't be practically possible, so no vibration can be thought of being in existence without damping. In a series of papers, recently DJO'Boy [3] have analyzed the damping of flexural vibration and Alisjahbana and Wangsadinata [4] discussed the realistic vibrational problem incorporating dynamic analysis of rigid roadway pavement under moving traffic loads. In the demand of modern science, a study dealing with damped vibrations of homogeneous isotropic rectangular plate of linearly varying thickness along one direction and resting on elastic foundation is presented employing classical plate theory. Various numerical techniques such as Frobenious method [5], finite difference method [6], simple polynomial approximation [7], Galerkin's method [8,9], Rayleigh-Ritz method [10-12], finite element method [13] and Chebyshev collocation method [14] etc, have been employed to analyzed the modes of vibration of plates with different geometries. The numerical methods require small interval size to obtain the results up to the desired accuracy due to round off and truncation errors at each step. Frobenious method results in the form of series and for the purpose of computation, series is truncated which leads incorporates some errors and the characteristics orthogonal polynomials requires an appreciable number of terms for plates of variable thickness. However Quintic splines interpolation technique has the capability of producing highly accurate results with minimum computational efforts for initial and boundary value problems. Therefore in the present paper, quintic spline method is used to obtain modes of vibration because a chain of lower-order approximations yields a better accuracy than a global higher-order approximations and natural boundary conditions can be incorporated easily [15]. The frequencies and deflection corresponding to the first three modes of vibrations are computed for various values of plate parameters such as taper constants, damping parameter and elastic foundations.

\section{Mathematical Formulation}

The plate under consideration is a rectangular isotropic plate of length 'a', breath ' $b$ ', thickness ' $h$ ' and density ' $\rho$ ', with resting on a winkler- type elastic foundation ' $k_{f}$ ' ' The plate is referred to rectangular cartesian co-ordinate $(\mathrm{x}, \mathrm{y}, \mathrm{z})$. The middle surface being $\mathrm{z}=0$ and the origin is at one of the corners of the plate. The differential equation which governs the transverse vibration of such plates is given by

$$
\begin{aligned}
& \nabla^{2}\left(D \nabla^{2} w\right)-(1-v)\left[\frac{\partial^{2} D}{\partial y^{2}} \frac{\partial^{2} w}{\partial x^{2}}-2 \frac{\partial^{2} D}{\partial x \partial y} \frac{\partial^{2} w}{\partial x \partial y}+\frac{\partial^{2} D}{\partial x^{2}} \frac{\partial^{2} w}{\partial y^{2}}\right]+\rho h \frac{\partial^{2} w}{\partial t^{2}}+K \frac{\partial w}{\partial t}+K_{f} w=0 \\
& \text { Where, } D=D(x, y)=\frac{E h^{3}(x, y)}{12\left(1-v^{3}\right)}
\end{aligned}
$$

Where $K$ the damping is constant, $K_{f}$ is the elastic foundation constant, $w(x, y, t)$ is the transverse deflection and $D$ is the flexural rigidity at any point in the middle plane of the plate. Let the two opposite edges $\mathrm{y}=0$ and $\mathrm{y}=\mathrm{b}$ of the plate be simply supported and thickness $h=h(x, y)$ varies linearly along the length i.e. in the direction of $x$-axis. Thus, ' $h$ ' is independent of y i.e. $h=h(x)$. For a harmonic solution, the deflection function $w$, satisfying the condition at $\mathrm{y}=0$ and $\mathrm{y}=\mathrm{b}$, is assumed

*Corresponding author: Robin, Department of Applied Science, Quantum Schoo of Technology, Roorkee, India, E-mail: robin1986sr@gmail.com

Received April 04, 2013; Accepted May 28, 2013; Published June 28, 2013

Citation: Robin, Rana US (2013) Damped Vibrations of Rectangular Plate of Variable Thickness Resting on Elastic Foundation: A Spline Technique. J Appl Computat Math 2: 130. doi:10.4172/2168-9679.1000130

Copyright: () 2013 Robin, et al. This is an open-access article distributed under the terms of the Creative Commons Attribution License, which permits unrestricted use, distribution, and reproduction in any medium, provided the original author and source are credited. 
Citation: Robin, Rana US (2013) Damped Vibrations of Rectangular Plate of Variable Thickness Resting on Elastic Foundation: A Spline Technique. J Appl Computat Math 2: 130. doi:10.4172/2168-9679.1000130

Page 2 of 6

$$
w(x, y, t)=W(x) \sin \frac{m \pi y}{b} e^{-\gamma t} \cos p t
$$

Where ' $\mathrm{p}$ ' is the circular frequency of vibration and ' $m$ ' is a positive integer. Thus Eq.(1) becomes

$$
\begin{aligned}
& {\left[h^{3} \frac{\partial^{4} W}{\partial x^{4}}+6 h^{2} \frac{\partial h}{\partial x} \frac{\partial^{3} W}{\partial x^{3}}+\left\{6 h\left(\frac{\partial h}{\partial x}\right)^{2}+3 h^{2} \frac{\partial^{2} h}{\partial x^{2}}-2 h^{3} \frac{m^{2} \pi^{2}}{b^{2}}\right\} \frac{\partial^{2} W}{\partial x^{2}}-6 h^{2} \frac{m^{2} \pi^{2}}{b^{2}} \frac{\partial h}{\partial x} \frac{\partial W}{\partial x}\right] \cos p t} \\
& +\left[\frac{m^{4} \pi^{4}}{b^{4}} h^{3}+3 v \frac{m^{2} \pi^{2}}{b^{2}}\left\{2 h\left(\frac{\partial h}{\partial x}\right)^{2}+h^{2} \frac{\partial^{2} h}{\partial x^{2}}\right\}\right] W \cos p t+K_{f} \frac{12\left(1-v^{2}\right)}{E} W \cos p t+ \\
& {\left[\frac{12\left(1-v^{2}\right) \rho h}{E}\left\{\left(\gamma^{2}-p^{2}\right) \cos p t+2 \gamma \sin p t\right\}+\frac{12\left(1-v^{2}\right)}{E} k\{-p \sin p t-\gamma \cos p t\}\right] W=0}
\end{aligned}
$$

Introducing the non-dimensional variables $H=\frac{h}{a}, X=\frac{x}{a}, \bar{W}=\frac{W}{a}$, $\beta^{2}=m^{2} \pi^{2}\left(\frac{a}{b}\right)^{2}$

Equation (3) reduces to

$$
\begin{aligned}
& {\left[H^{3} \frac{\partial^{4} \bar{W}}{\partial X^{4}}+6 H^{2} \frac{\partial H}{\partial X} \frac{\partial^{3} \bar{W}}{\partial X^{3}}+\left\{6 H\left(\frac{\partial H}{\partial X}\right)^{2}+3 H^{2} \frac{\partial^{2} H}{\partial X^{2}}-2 H^{3} \beta^{2}\right\} \frac{\partial^{2} \bar{W}}{\partial X^{2}}-6 H^{2} \beta^{2} \frac{\partial H}{\partial X} \frac{\partial \bar{W}}{\partial X}\right] \cos p t} \\
& +\left[\beta^{4} H^{3}+3 v \beta^{2}\left\{2 H\left(\frac{\partial H}{\partial X}\right)^{2}+H^{2} \frac{\partial^{2} H}{\partial X^{2}}\right\}\right] \bar{W} \cos p t+K_{f} \frac{12\left(1-v^{2}\right)}{E} \bar{W} \cos p t+ \\
& {\left[\frac{12\left(1-v^{2}\right) \rho H}{E}\left\{\left(\gamma^{2}-p^{2}\right) \cos p t+2 \gamma \sin p t\right\}+\frac{12\left(1-v^{2}\right)}{E} k\{-p \sin p t-\gamma \cos p t\}\right] \bar{W}=0}
\end{aligned}
$$

Substituting $H=H_{0}(1-\alpha X)$, where $H_{0}=(H)_{X=0}$ and ' $\alpha$ ' is the taper constant due to linearly varying thickness of plate, and equating the coefficient of $\sin (p t)$ and $\cos (p t)$ independently to zero, following equation is formed

$$
A_{0} \frac{\partial^{4} \bar{W}}{\partial X^{4}}+A_{1} \frac{\partial^{3} \bar{W}}{\partial X^{3}}+A_{2} \frac{\partial^{2} \bar{W}}{\partial X^{2}}+A_{3} \frac{\partial \bar{W}}{\partial X}+A_{4} \bar{W}=0
$$

Where

$$
\begin{aligned}
& A_{0}=(1-\alpha X)^{4}, A_{1}=-6 \alpha(1-\alpha X)^{3}, A_{2}=6 \alpha^{2}(1-\alpha X)^{2}-2 \beta^{2}(1-\alpha X)^{4}, A_{3}=6 \alpha \beta^{2}(1-\alpha X)^{3} \\
& A_{4}=\beta^{4}(1-\alpha X)^{4}-6 \alpha^{2} \beta^{2} v(1-\alpha X)^{2}-\left\{D_{K}{ }^{2} I^{* 2}+\Omega^{2} I^{*}(1-\alpha X)^{2}-E_{f}(1-\alpha X)^{3} C^{*}\right\} \\
& D_{K}=\frac{3\left(1-v^{2}\right) K^{2}}{E \rho}, I^{*}=\frac{1}{H_{0}{ }^{2}}, C^{*}=\frac{1}{H_{0}{ }^{3}}, E_{f}=\frac{12\left(1-v^{2}\right) a}{E_{0}}, \Omega^{2}=\frac{12\left(1-v^{2}\right) a^{2} \rho p^{2}}{E},
\end{aligned}
$$

$\Omega, D_{K}, E_{f}$ are frequency parameter, damping parameter and elastic foundation parameter respectively.

The solution of Eq.(5) together with boundary conditions at the edge $\mathrm{x}=0$ and $\mathrm{x}=1$ constitutes a two-point boundary value problem. As the PDE has several plate parameters, therefore it becomes quite difficult to find it's exact solution. Keeping this in mind, complex for the purpose of computation, the quintic spline interpolation technique, is used. Let $\mathrm{f}(\mathrm{x})$ be a function with continuous derivatives in the range $[0,1]$ and interval $[0,1]$ be divided into ' $n$ ' subintervals by means of points $X_{i}$ such that $0=X_{0}<X_{1}<X_{2}<\ldots<X_{n}=1$. Where $\Delta X=1 / n, X_{i}=i \Delta X(i=0,1,2, \ldots, n)$. Let the approximating function $\bar{W}(X)$ for the $\mathrm{f}(\mathrm{x})$ be a quintic spline with the following properties:

(i) $\bar{W}(X)$ is a quintic polynomial in each interval $\left(X_{k}, X_{k+1}\right)$.

(ii) $\bar{W}(X)=F\left(X_{k}\right), k=0,1,2, \ldots, n$.

(iii) $\frac{\partial \bar{W}}{\partial X}, \frac{\partial^{2} \bar{W}}{\partial X^{2}}, \frac{\partial^{3} \bar{W}}{\partial X^{3}}$ and $\frac{\partial^{4} \bar{W}}{\partial X^{4}}$ are continuous.

In view of above axioms, the quintic spline takes the form

$$
\bar{W}(X)=a_{0}+\sum_{i=0}^{4} a_{i}\left(X-X_{0}\right)^{i}+\sum_{j=0}^{n-1} b_{j}\left(X-X_{j}\right)_{*}^{5}
$$

Where $\left(X-X_{j}\right)_{*}=\left\{\begin{array}{c}0, \quad \text { if } X \leq X_{j} \\ \left(X-X_{j}\right), \text { if } X>X_{j}\end{array}\right.$

and $a_{0}, a_{1}, a_{2}, a_{3}, a_{4}, b_{n-1}$ are $(\mathrm{n}+5)$ unknown constants. Thus for the satisfaction at the $\mathrm{n}^{\text {th }}$ knot, Eq.(6) reduced to

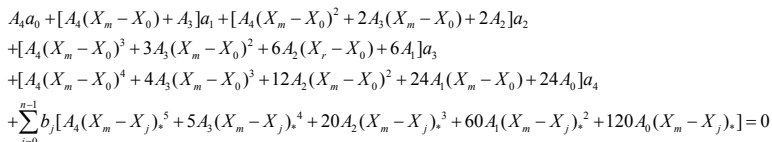

For $\mathrm{m}=0(1) \mathrm{n}$, above system contains $(\mathrm{n}+1)$ homogeneous equation with $(n+5)$ unknowns, ai, $i=0(1) 4, b j, j=0,1,2, \ldots,(n-1)$, and can be represented in matrix form as $[\mathrm{A}]\{\mathrm{B}\}=\{0\}$

Where $[A]$ is a matrix of order $(n+1) \times(n+5)$ while $\{B\}$ and $\{0\}$ are column matrices of order $(\mathrm{n} \times 5)$.

\section{Boundary Conditions and Frequency Equation}

The following two cases of boundary conditions have been considered:

(i) (c-ss-c-ss): clamped at both the edge $\mathrm{X}=0$ and $\mathrm{X}=1$.

(ii) (c-ss-ss-ss): clamped at $\mathrm{X}=0$ and simply supported at $\mathrm{X}=1$.

The relations that should be satisfied at clamped and simply supported are

$W=\frac{\partial W}{\partial X}=0 ; W=\frac{\partial^{2} W}{\partial X^{2}}=0 ;$ respectively.

Applying the boundary conditions c-ss-c-ss to the displacement function by Eq. (9) one obtains a set of four homogeneous equations in terms of $(n+5)$ unknown constants which can be written as

$\left[\mathrm{B}^{\mathrm{cc}}\right]\{\mathrm{B}\}=\{0\}$

Where $\mathrm{B}^{\mathrm{cc}}$ is a matrix of order $4 \times(\mathrm{n}+5)$. Therefore the Eq. (7) together with the Eq. (10) gives a complete set of $(n+5)$ homogeneous equations having $(n+5)$ unknowns which can be written as

$$
\left[\frac{A}{B^{c c}}\right]\{B\}=\{0\}
$$

For a non-trival solution of Eq. (11), the characteristic determinant must vanish, i.e.

$$
\left|\frac{A}{B^{c c}}\right|=0
$$

Similarly for (c-ss-ss-ss) plate the frequency determinant can be written as

$$
\left|\frac{A}{B^{s s}}\right|=0 \text {, Where Bss is a matrix of order } 4 \times(\mathrm{n}+5)
$$

\section{Numerical Results and Discussion}

In the present paper, first three frequency modes of vibration have been computed for the above mentioned two boundary conditions for different values of foundation parameter $E_{f}=0.0(0.005) 0.02$, damping parameter $D_{k}=0.0(0.01) 0.04$ and taper parameter $\alpha=0.0(0.1) 0.4$ for Poisson ratio's $v=0.3$, thickness of plate $\mathrm{h}=0.03$ and aspect ratio a/ $\mathrm{b}=0.25$. The numerical method provides approximate values therefore in order to minimize the error, there is an urgent need to determine the optimum size of interval length $\Delta \mathrm{X}$. In the present problem, a computer 
Citation: Robin, Rana US (2013) Damped Vibrations of Rectangular Plate of Variable Thickness Resting on Elastic Foundation: A Spline Technique. J Appl Computat Math 2: 130. doi:10.4172/2168-9679.1000130

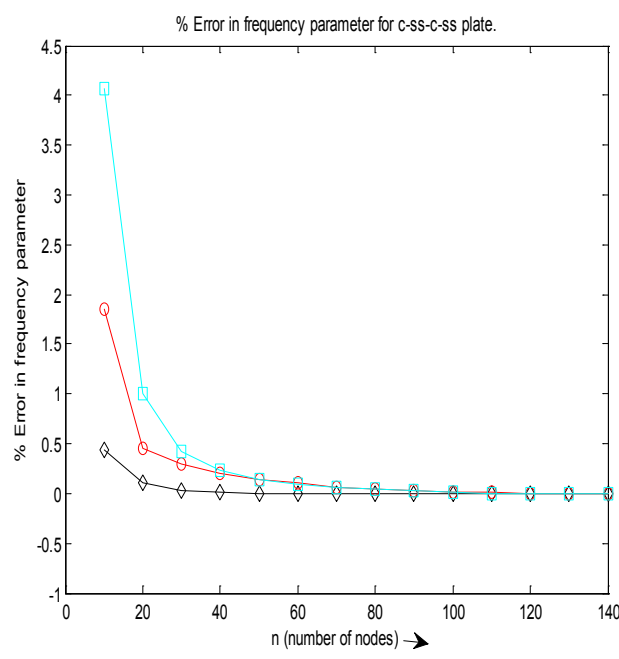

(a)

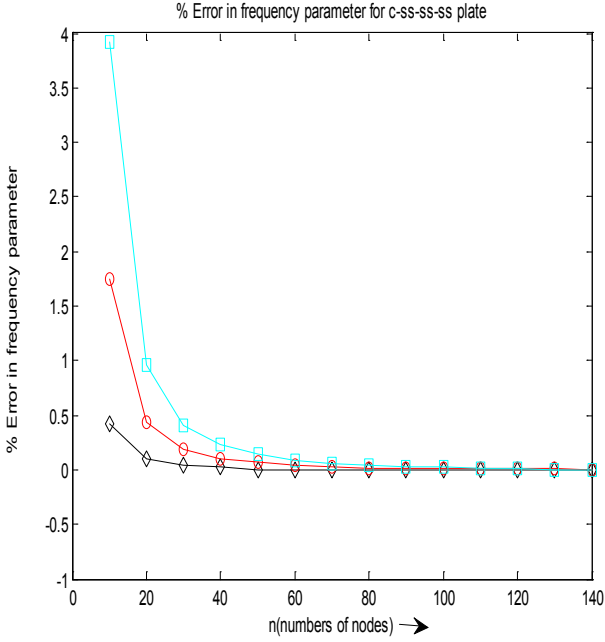

(b)

Figure 1: percentage error in frequency parameter $\Omega$ : (a) c-ss-c-ss plate (b) c-ss-ss-ss plate, for $a / b=0.25, \alpha=0.0, D_{k}=0.0, P e r c e n t a g e ~ e r r o r=\left[\left(\Omega_{n}-\Omega_{140}\right) /\right.$ $\left.\Omega_{140}\right] \times 100 ; n=10(10) 140$

\begin{tabular}{|c|c|c|c|c|c|c|}
\hline & $h=0.03$ & $v=0.3$ & $m=1$ & $a / b=0.25$ & $D k=0.0$ & $E_{f}=0.0$ \\
\hline & \multicolumn{3}{|c|}{ c-ss-c-ss plate } & \multicolumn{3}{|c|}{ c-s-ss-ss plate } \\
\hline taper parameter $(\alpha)$ & mode1 & mode2 & mode3 & mode1 & mode2 & mode3 \\
\hline 0 & 0.6816 & 1.8643 & 3.6432 & 0.4766 & 1.515 & 3.1449 \\
\hline 0.1 & 0.6471 & 1.7699 & 3.4587 & 0.4584 & 1.4442 & 2.9916 \\
\hline 0.2 & 0.6117 & 1.6729 & 3.2689 & 0.4396 & 1.3715 & 2.8341 \\
\hline 0.3 & 0.5752 & 1.5727 & 3.0729 & 0.42 & 1.2963 & 2.6741 \\
\hline 0.4 & 0.5373 & 1.4686 & 2.8692 & 0.3995 & 1.2184 & 2.5025 \\
\hline
\end{tabular}

Table 1a: Values of frequency parameter $\Omega$ for different values of taper constant $\alpha$.

\begin{tabular}{|c|c|c|c|c|c|c|}
\hline & $h=0.03$ & $v=0.3$ & $m=1$ & $a / b=0.25$ & Dk $=0.0025$ & $E_{f}=0.0$ \\
\hline & \multicolumn{3}{|c|}{ c-ss-c-ss plate } & \multicolumn{3}{|c|}{ c-ss-ss-ss plate } \\
\hline taper parameter $(\alpha)$ & mode1 & mode2 & mode3 & mode1 & mode2 & mode3 \\
\hline 0 & 0.6765 & 1.8624 & 3.6422 & 0.4692 & 1.5128 & 3.1438 \\
\hline 0.1 & 0.6411 & 1.7677 & 3.4576 & 0.4498 & 1.4416 & 2.9903 \\
\hline 0.2 & 0.6046 & 1.6703 & 3.2676 & 0.4293 & 1.3683 & 2.8325 \\
\hline 0.3 & 0.5666 & 1.5695 & 3.0713 & 0.4076 & 1.2924 & 2.6695 \\
\hline 0.4 & 0.5266 & 1.4647 & 2.8671 & 0.3841 & 1.2135 & 2.5001 \\
\hline
\end{tabular}

Table 1b: Values of frequency parameter $\Omega$ for different values of taper constant $\alpha$.

program was developed and executed for $n=10(10) 150$ and observed that no consistent improvement in results while $\mathrm{n} \geq 140$ for clarity (Figures $1 \mathrm{a}$ and $1 \mathrm{~b}$ ). Therefore the results are obtained for $\mathrm{n}=140$ and depicted through table and graphs. It has also been observed that the frequencies for c-ss-c-ss plates are more than the frequencies of c-ssss-ss plates for the same set of values of other parameters. Tables 1a-1c and Figures $2 \mathrm{a}-2 \mathrm{c}$ show the behavior of frequency parameter $\Omega$ with the increasing value of taper constant $(\alpha)$ for the fixed value of damping parameter $D_{k}=0.0,0.0025$, and foundation parameter $E_{f}=0.0,0.01$ for first three modes of vibration of c-c and c-ss plate, it is observed that the frequency parameter $\Omega$ decreases with the increasing values of taper parameter $a$. Tables $2 a-2 b$ and Figures $3 a$ and $3 b$, provide the inference of damping parameter $D_{k}$ on frequency parameter $\Omega$ for two values of and foundation parameter $E_{f}=0.0$, and 0.01 respectively, for the fixed value of taper parameter $\alpha=0.4$. It is observed that the frequency parameter decreases with the increases of damping parameter $D_{k}$

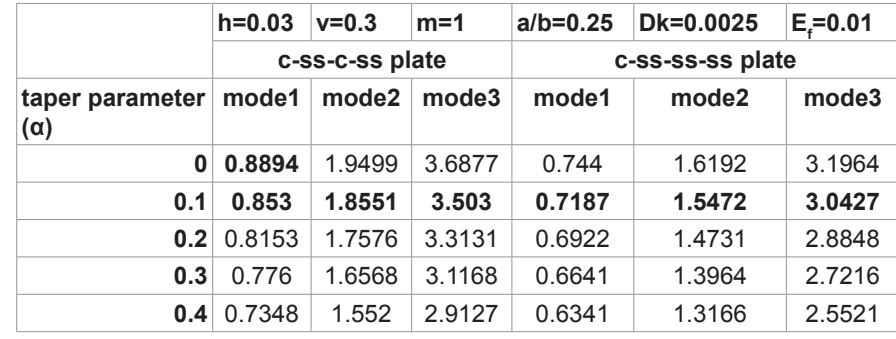

Table 1c: Values of frequency parameter $\Omega$ for different values of taper constant $\alpha$

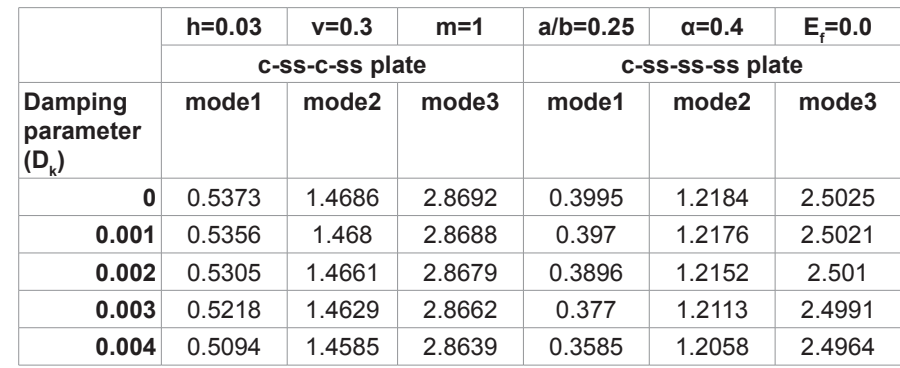

Table 2a: Values of frequency parameter $\Omega$ for different values of damping parameter $D_{k}$

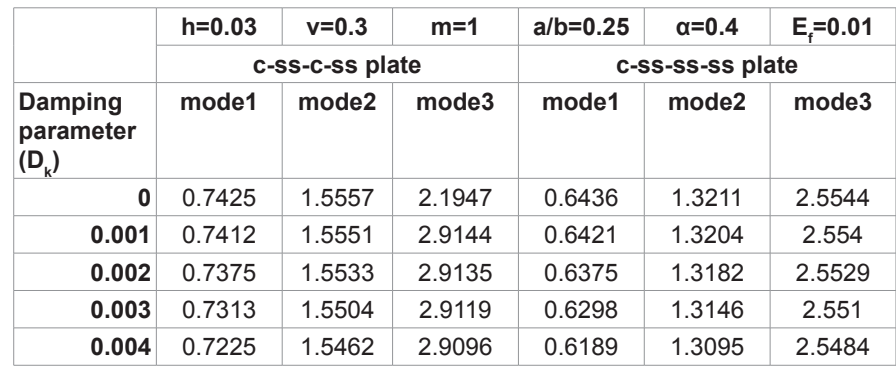

Table 2b: Values of frequency parameter $\Omega$ for different values of damping parameter $D_{k}$. 
Citation: Robin, Rana US (2013) Damped Vibrations of Rectangular Plate of Variable Thickness Resting on Elastic Foundation: A Spline Technique. J Appl Computat Math 2: 130. doi:10.4172/2168-9679.1000130

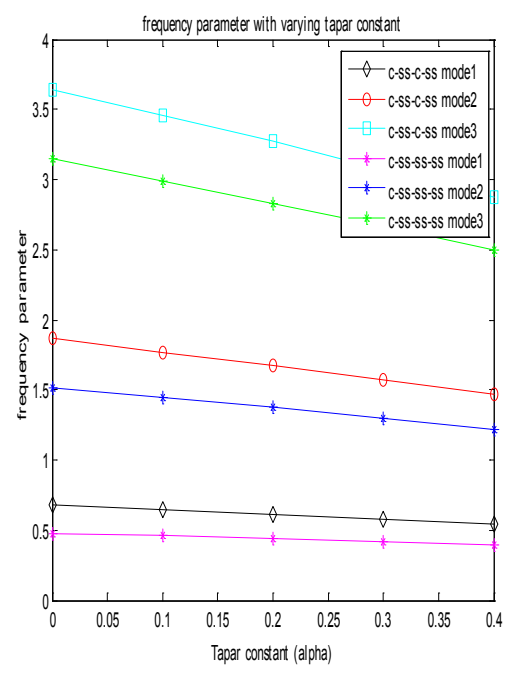

(a)

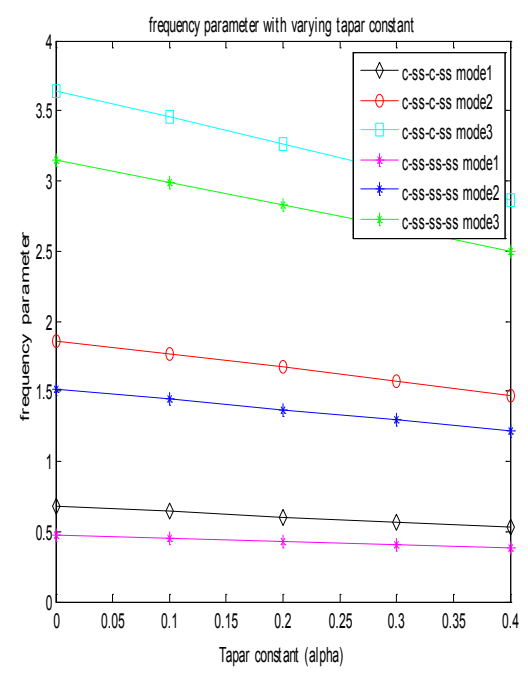

(b)

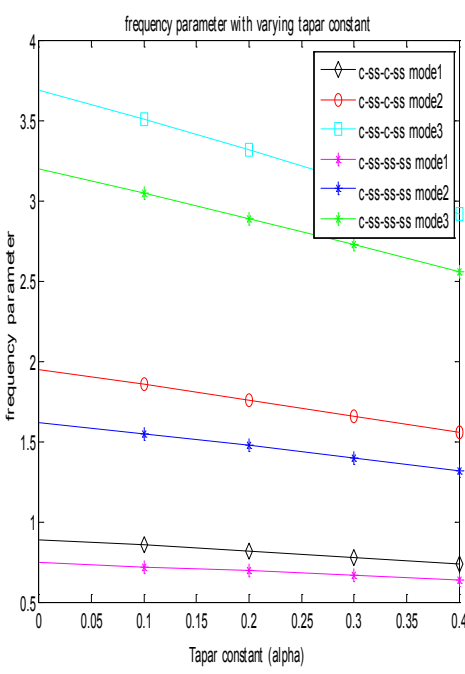

(c)

Figure 2: Frequency parameter for clamped, simply supported plates: (a) modes for $D_{k}=0.0, E_{f}=0.0$; (b) modes for $D_{k}=0.0025, E_{f}=0.0$; (c) modes for $D_{k}=0.0025$, $E_{f}=0.01$.

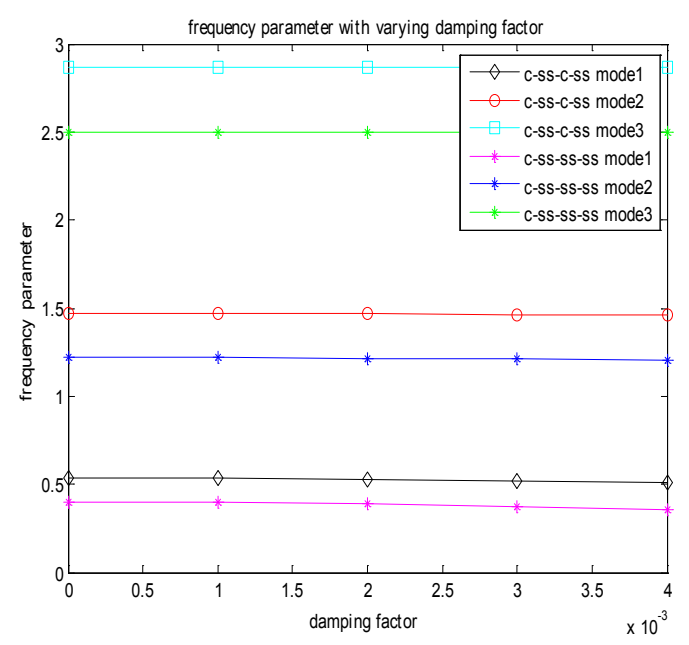

(a)

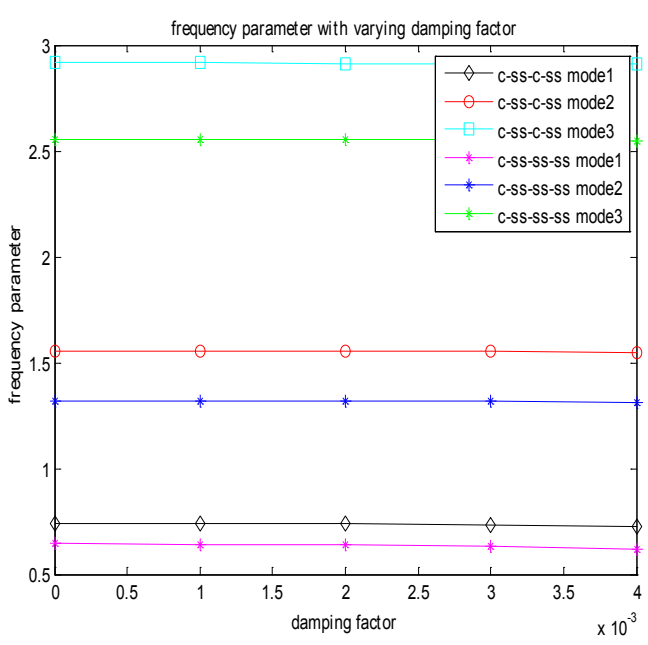

(b)

Figure 3: Frequency parameter for clamped, simply supported plates: (a) modes for $\alpha=0.0, E_{f}=0.0$; (b) modes for $\alpha=0.4$, $E_{f}=0.01$.

\begin{tabular}{|c|c|c|c|c|c|c|}
\hline & $h=0.03$ & $v=0.3$ & $m=1$ & $a / b=0.25$ & $\alpha=0.4$ & $D_{k}=0.0$ \\
\hline & \multicolumn{3}{|c|}{ c-ss-c-ss plate } & \multicolumn{3}{|c|}{ c-ss-ss-ss plate } \\
\hline Foundation & mode1 & mode2 & mode3 & mode1 & mode2 & mode3 \\
\hline 0 & 0.5373 & 1.4686 & 2.8692 & 0.3995 & 1.2184 & 2.5025 \\
\hline 0.005 & 0.6481 & 1.5128 & 2.892 & 0.5357 & 1.2707 & 2.5286 \\
\hline 0.01 & 0.7425 & 1.5557 & 2.9147 & 0.6436 & 1.3211 & 2.5544 \\
\hline 0.015 & 0.8261 & 1.5975 & 2.9373 & 0.7357 & 1.3696 & 2.5799 \\
\hline 0.02 & 0.9019 & 1.6383 & 2.9596 & 0.8175 & 1.4165 & 2.6052 \\
\hline
\end{tabular}

Table 3a: Values of frequency parameter $\Omega$ for different values of foundation parameter $\mathrm{E}_{f}$

\begin{tabular}{|c|c|c|c|c|c|c|}
\hline & $h=0.03$ & $v=0.3$ & $m=1$ & $a / b=0.25$ & $\alpha=0.4$ & $D_{k}=0.0025$ \\
\hline & \multicolumn{3}{|c|}{ c-ss-c-ss plate } & \multicolumn{3}{|c|}{ C-ss-ss-ss plate } \\
\hline Foundation & mode1 & mode2 & mode3 & mode1 & mode2 & mode3 \\
\hline 0 & 0.5266 & 1.4647 & 2.8671 & 0.384 & 1.2135 & 2.5011 \\
\hline 0.005 & 0.6392 & 1.5089 & 2.89 & 0.5242 & 1.2661 & 2.5262 \\
\hline 0.01 & 0.7348 & 1.552 & 2.9127 & 0.6341 & 1.3166 & 2.5521 \\
\hline 0.015 & 0.8191 & 1.5939 & 2.9353 & 0.7274 & 1.3653 & 2.5776 \\
\hline 0.02 & 0.8955 & 1.6347 & 2.9577 & 0.8099 & 1.4123 & 2.603 \\
\hline
\end{tabular}

Table 3b: Values of frequency parameter $\Omega$ for different values of foundation parameter $\mathrm{E}_{f}$ 
Citation: Robin, Rana US (2013) Damped Vibrations of Rectangular Plate of Variable Thickness Resting on Elastic Foundation: A Spline Technique. J Appl Computat Math 2: 130. doi:10.4172/2168-9679.1000130

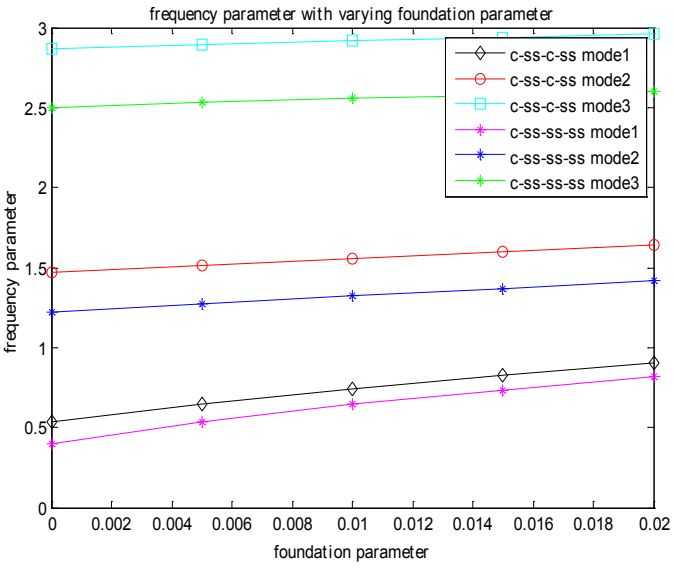

(a)

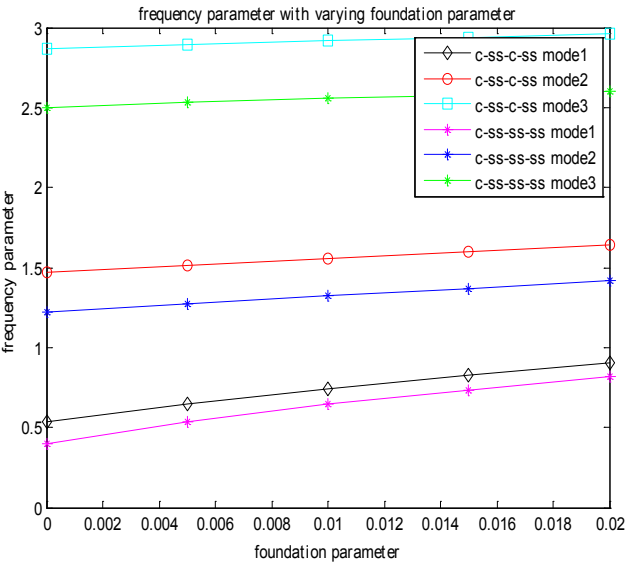

(b)

Figure 4: Frequency parameter for clamped, simply supported plates: (a) modes for $\alpha=0.04, D_{k}=0.0$; (b) modes for $\alpha=0.4, D_{k}=0.0025$.

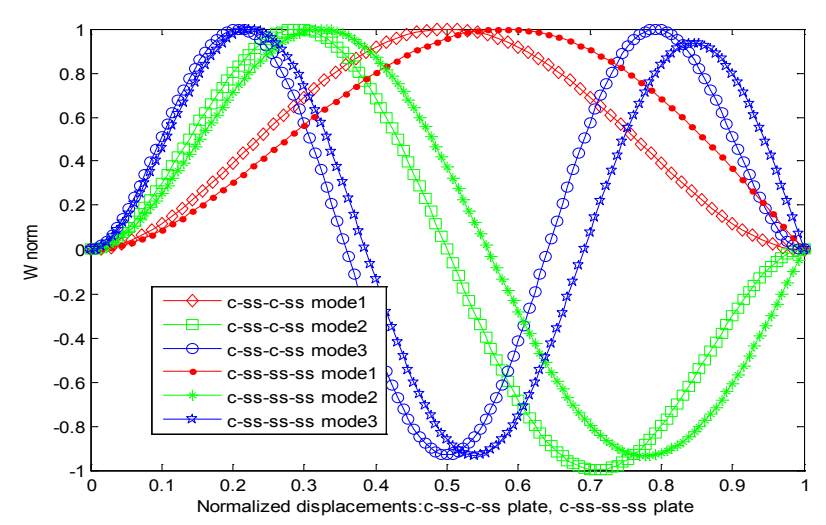

Figure 5: Normalized displacements for clamped and simply supported plates, for $a / b=0.025 ; h=0.03, D_{k}=0.0, E f=0.0$.

and the rate of decreases with the increases in the value of damping parameter $D_{k}$ for c-ss plates is higher than that for c-c plate keeping all other plate parameter fixed. Tables $3 \mathrm{a}$ and $3 \mathrm{~b}$ and Figures $4 \mathrm{a}$ and $4 \mathrm{~b}$ show the increase in frequency parameter $\Omega$ verses foundation parameter $E_{f}$ for two different value of damping parameter $D_{k}=0.0$, 0.0025 , for the fixed value of taper parameter $\alpha=0.4$. It is noticed that the frequency parameter $\Omega$ increases continuously with the increasing value of foundation parameter $E_{f}$ for c-ss-c-ss and c-ss-ss-ss plates, whatever be the value of other plate parameters. It is found that the rate of increases of frequency parameter $\Omega$ for c-ss-ss-ss plate is higher than c-ss-c-ss plate for three modes. The normalized displacements for the two boundary conditions c-ss-c-ss and c-ss-ss-ss, considered in this paper are shown in Figure 5. The plate thickness varies linearly in $\mathrm{X}$-direction and the plate is considered resting on elastic foundations. Figure 5 Shows the transverse displacements for the first three modes. The nodal lines are seen to shift towards the edge, i.e. $X=1$ as the boundary conditions change from c-ss-c-ss to c-ss-ss-ss for all the three transverse modes. The slope of the normalized displacement curves on both edges for c-ss-c-ss condition, are nearly zero which indicates the correctness of the solution.

\section{Conclusion}

In the present study results are computed using MATLAB within the permissible range of parameters up to the desired accuracy $\left(10^{-8}\right)$, which validates the actual phenomenon of vibrational problem. Variation in thickness, elastic foundation and damping parameter are of great interest since it provides reasonable approximation to linear vibrations. Thus the present study may be useful for design engineers especially in rigid roadway pavement under moving traffic loads.

\section{References}

1. AW Leissa (1969) Vibration of Plates, NASA SP-160, Government Printing Office, Washington, DC.

2. Lal R, Gupta US, Reena (1997) Quintic splines in the study of transverse vibrations of non-uniform orthotropic rectangular plates. Journal of Sound and Vibration 207: 1-13.

3. O'Boy DJ, Krylov VV (2011) Damping of flexural vibrations in circular plates with tapered central holes. Journal of Sound and Vibration 330: 2220-2236.

4. Alisjahbana SW, Wangsadinata W (2012) Dynamic analysis of rigid roadway pavement under moving traffic loads with variable velocity. Interaction and Multiscale Mechanics 5: 105-114.

5. Lal R (1979) Vibrations of Elastic plates of Variable Thickness, PhD Thesis University of Roorkee.

6. Singh B, Hassan SM, Lal J (1996) Some numerical experiments on high accuracy fast direct finite difference methods for elliptic problem. Communications in Numerical Methods in Engineering 12: 631-641.

7. Gupta US, Lal R, Jain SK (1990) Effect of elastic foundation on axisymmetric vibration of polar orthotropic circular plates of variable thickness. Journal of Sound and Vibration 139: 503-513.

8. Avalos DR, Hack H, Laura PAA (1982) Galerkin's method and axisymmetric vibrations of polar orthtropic circular plates. AIAA Journal 20: 1626-1628.

9. Ratko M (2005) Transverse vibration and instability of an eccentric rotating circular plate. Journal of Sound and Vibration 280: 467-478.

10. Singh B, Saxena V (1996) Transverse vibration of a circular plate with unidirectional quadratic thickness variations. International Journal of Mechanical Science 38: 423-430.

11. Gupta US, Ansari AH (2003) Transverse vibration of polar orthotropic parabolically tapered circular plates. Indian Journal of Pure and Applied Mathematics 34: 819-830.

12. Singh B, Chakraverty S (1994) Use of characteristic orthogonal polynomials 
Citation: Robin, Rana US (2013) Damped Vibrations of Rectangular Plate of Variable Thickness Resting on Elastic Foundation: A Spline Technique. J Appl Computat Math 2: 130. doi:10.4172/2168-9679.1000130

in two dimensions for transverse vibration of elliptical and circular plates with variable thickness. Journal of Sound and Vibration 173: 289-299.

13. Chen DY, Ren BS (1998) Finite element analysis of the lateral vibration of thin annular and circular plates with variable thickness. ASME Journal of Vibration and acoustics 120: 747-752.
14. Lal R, Gupta US, Goel C (2001) Chebyshev polynomials in the study of transverse vibration of non-uniform rectangular orthotropic plates. The Shock and Vibration Digest 33: 103-112.

15. Bickley WG (1968) Piecewise cubic interpolation and two point boundary problems. The Computer Journal 11: 206-208. 